\title{
Pilot study of a newly developed expiratory positive airway pressure device
}

\author{
Irina Dralyuk ${ }^{1,2}$, Inderpal Randhawa ${ }^{1,2,3,4 *}$, Christopher Mann ${ }^{3}$, Arthur Gelb ${ }^{3}$, Maged Tanios ${ }^{3}$, Tricia Morphew and Eliezer Nussbaum $^{1,2}$ \\ ${ }^{1}$ Division of Pediatric Pulmonary Medicine, University of California, Irvine, CA, USA \\ ${ }^{2}$ Miller Children's and Women's Hospital Long Beach, Long Beach, CA, USA \\ ${ }^{3}$ Long Beach Memorial Medical Center, Long Beach, CA, USA \\ ${ }^{4}$ The Translational Pulmonary \& Immunology Research Center, Long Beach, CA, USA \\ ${ }^{5}$ Morphew Consulting LLC, Bothell, WA, USA
}

\begin{abstract}
Background: Obstructive sleep apnea [OSA], a sleep-related breathing disorder that affects millions of people worldwide, is the result of partial or complete airflow obstruction that occurs during sleep. Oropharyngeal muscle relaxation during sleep can result in soft tissue collapse, with subsequent airflow impedance. During episodes of airflow obstruction, oxygen levels can drop precipitously, inducing a neurological arousal. Carbon dioxide build up interrupts sleep which triggers the respiratory drive for resumption of coordinated breathing. Complete wakefulness is rarely achieved during these episodes, but sleep quality is severely compromised. If left untreated, OSA may increase the risk of cardiovascular disease, arrhythmia, hypertension, stroke, diabetes, depression, obesity, and intellectual decline. Compliance with and tolerance of existing devices particularly the Continuous Positive Airway Pressure (CPAP) machine remains poor.
\end{abstract}

Objectives: The present pilot study evaluates the utility of a newly developed mechanical nasal expiratory positive airway pressure [EPAP] device in OSA management.

Method: A total of 19 adult subjects were recruited from two accredited sleep study centers based on a pre-existing, polysomnographic diagnosis of OSA with mild to moderate apnea-hypopnea index [AHI] scores. Sixteen subjects completed the study and underwent a standard polysomnography [PSG] procedure while wearing the nasal study device. The device consists of two cylindrical bodies with a ball-valve mechanism that creates airflow resistance during expiration, allowing for pneumatic splinting. Resulting data were compared to baseline PSG results, performed in the same sleep center as the study PSG.

Results: Total apneas and hypopneas, respective indices for both, the cumulative apnea-hypopnea index, mean and minimum oxygen saturations during sleep, average and maximum heart rate during sleep, total desaturations and the desaturation index were evaluated and showed overall improvement. In the initial 6 patients, no significant improvement was observed in the overall indices; modifications were made to the device to minimize leak, and the subsequent 6 patients demonstrated a statistically significant improvement in obstructive indices and the minimum oxygen saturation achieved during sleep.

Discussion: Thus far, 16 of 19 subjects have successfully completed a standard PSG with the nasal device. One subject aborted the study early due to emotional instability unrelated to the use of the device. Another subject with particularly small nasal passages reported that the device felt uncomfortably snug and aborted the study early as well. The remaining subjects tolerated the study well, without experiencing any appreciable side effects, and most patients have found the device to be comfortable. Ongoing improvements to the device have been implemented based on subject feedback in order to optimize comfort and efficacy of the device.

Conclusion: This pilot study demonstrates the feasibility of using the EPAP device in patients with OSA. The device has been well tolerated by study subjects, has been reported to be comfortable, and standard sleep variables can be acquired with the device in place. Initial findings on the most updated model indicate improvement in AHI scores, oxygen saturation rates, and heart rate measured during sleep.

\begin{abstract}
Abbreviations: OSA: Obstructive sleep apnea; CPAP: Continuous Positive Airway Pressure; AHI: Apnea-Hypopnea Index; EPAP: Expiratory Positive Airway Pressure; PSG: Polysomnography; AASM: American Academy of Sleep Medicine; LSAT: Lowest Oxygen Saturation; PEEP: Positive End-Expiratory Pressure; ODI: Oxygen Desaturation Index.
\end{abstract}

\section{Introduction}

Obstructive sleep apnea [OSA], a sleep-related breathing disorder that affects millions of people worldwide, is the result of partial or complete airflow obstruction that occurs primarily during sleep [1]. Oropharyngeal muscle relaxation during sleep can result in soft tissue collapse, with subsequent airflow impedance [2]. During episodes of airflow obstruction, oxygen levels can drop precipitously, inducing a neurological arousal. Carbon dioxide build up interrupts sleep which triggers resumption of coordinated breathing [3,4]. Complete wakefulness is rarely achieved during these episodes, but sleep quality is severely compromised. If left untreated, OSA may increase the risk of cardiovascular disease, arrhythmia, hypertension, stroke, diabetes, depression, obesity, and intellectual decline $[5,6]$.

Positive airway pressure (PAP), initially described by Sullivan in 1981, produces pneumatic splinting of the upper airway, thereby reducing the effects of airway collapse [7-10]. It remains the gold

${ }^{*}$ Correspondence to: Inderpal Randhawa, The Translational Pulmonary and Immunology Research Center (TPIRC) 701 E. 28th \#419, Long Beach, CA 90806, USA, E-mail: docrandhawa@gmail.com

Received: April 08, 2019; Accepted: April 23, 2019; Published: April 25, 2019 
standard therapy for moderate to severe OSA. Unfortunately, there are significant issues with therapeutic adherence due to associated discomfort with PAP equipment including noise, pressure over facial and nasal tissue, and the inability of some patients to tolerate the treatment modality as it disrupts sleep altogether. Weaver et al found 46$83 \%$ of patients with OSA have been reported to be non-adherent with CPAP treatment [11]. Due to such a high proportion of patients who are unable to benefit from PAP therapy, efforts to produce alternative treatment options for OSA have been explored.

A review of the currently existing literature on adjunct therapies available has demonstrated an important role of nasal expiratory positive airway pressure in the treatment of OSA $[8,12]$. The initial use of passive EPAP application by Mahadevia et al in 1983 showed a significant improvement in apnea index and oxygen desaturation index [13]. More recent studies by Berry et al. [2], Kryger et al. [3] and Colrain et al. [11] have shown a substantial reduction of the AHI and oxygen desaturation indices in patients with varying severity of sleep apnea. Reviewed the existing published data on nasal expiratory positive airway pressure devices in a meta-analysis and found that nasal EPAP reduced AHI scores by $53.2 \%$, ODI scores by $41.5 \%$, and improved Lowest Oxygen Saturation [LSAT] scores by 3 oxygen saturation points. However, no nasal expiratory device has reached widespread therapeutic use in OSA.

Nasal expiratory devices result in positive end-expiratory pressure [PEEP] results in higher end expiratory lung volumes which in turn increases the longitudinal traction on the pharynx, thus making it less prone to collapse. Additionally, the improved dilation of the upper airway by EPAP may last throughout the expiratory phase. In total effect, the mild elevation of $\mathrm{PaCO}^{2}$ approximated by end tidal $\mathrm{CO}^{2}$ secondary to hypoventilation, or decreased rate of breathing, leads to an increased respiratory drive to the upper airway [6]. Given the potential benefit, nasal expiratory devices lack common use due to convenience and comfort.

A new device has been developed to alleviate the deleterious effects of OSA in patients who are unable to tolerate CPAP treatment or desire a convenient, portable therapeutic option. The product being tested is a potential alternative characterized by convenient nasal application without associated equipment or external pressure and compressor support. The medical grade plastic used to manufacture this product is safe for human use, has not been associated with any allergic or irritation symptoms, has no sharp edges or protrusions that could potentially cause discomfort or distress, and is very easy to clean and maintain. Furthermore, it is compact and can be used while traveling, unlike the large, unwieldy equipment associated with CPAP therapy. This device, if found to be therapeutic, would offer a much more comfortable, disposable, safe way to alleviate the burden of OSA. The present pilot study evaluates the utility of a newly developed mechanical nasal expiratory positive airway pressure device in OSA management.

\section{Methods}

\section{Study subjects}

Nineteen patients were recruited from two separate accredited sleep centers participating in the study. The electronic databases of each clinic were screened for potential subjects who met inclusion/exclusion criteria. Baseline demographic characteristics, additional preexisting medical conditions and previous PSG results were assessed.

Subjects were excluded if they had nasal deformities or nasal occlusion, severe nasal allergies, rhinitis or moderate nasal congestion, acute upper respiratory inflammation or infection, or perforation of the eardrum. In addition subjects were excluded if they had co-morbid sleep disorders, immune deficiency or were taking immunosuppressive agents, used hypnotic medications for insomnia, had an uncontrolled or serious illness, including but not limited to severe breathing disorders, including hypercapnic respiratory failure, severe nocturnal arterial oxygen desaturation, respiratory muscle weakness, bullous lung disease, bypassed upper airway, pneumothorax, pneumomediastinum, etc., severe heart disease, or pathologically low blood pressure. Female subjects of childbearing age were excluded if they were pregnant or intending to become pregnant.

Adult patients with a pre-existing diagnosis of mild to severe OSA based on a previously diagnosed apnea-hypopnea index score between 5 and 45 obtained within 24 months of the screening visit were eligible for recruitment. The ability to tolerate using the device during prior to the initiation of the study was also required for participation.

\section{Study design}

Each patient served as his or her own control. Patients underwent standard PSG study at one of the two participating sleep study centers, with the administration of the EPAP device prior to initiation of the PSG. Comprehensive recordings of the biophysical changes that occurred during sleep were collected throughout the course of the night. Standard sleep parameters, including an AHI index, were subsequently calculated based on the study results. The interpretation of all polysomnograms was conducted by a board-certified sleep specialist who was completely blinded to the first baseline PSG results and was not allowed to make comparative analysis when interpreting the second PSG during which subjects utilized the device. The trial was approved by the University of California Irvine Institutional Review Board, and written informed consent was obtained from patients in accordance with standard requirements.

The Apnea-Hypopnea Index [AHI] is commonly used to describe the severity of OSA, with scores of 5-15 indicating mild disease, 15-30 describing moderate disease, and scores above 30 being indicative of severe disease. The Adult OSA Task Force of the American Academy of Sleep Medicine [AASM] defines the criteria for OSA diagnosis as the presence of 15 or more obstructive events per hour during polysomnography [PSG], or 5 events per hour and the presence of any of the following: unintentional sleep episodes during wakefulness; daytime sleepiness; unrefreshing sleep; fatigue; insomnia; waking up breath holding, gasping, or choking; or the bed partner describing loud snoring, breathing interruptions, or both during the patient's sleep [9].

\section{Device design}

The nasal airway pressure device (Figure 1) is a single unit that consists of two small cylindrical bodies attached to each other by a

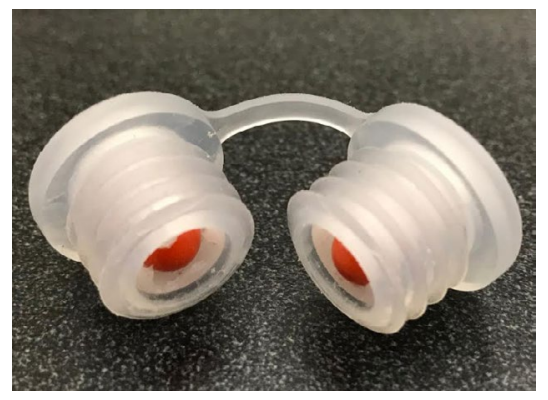

Figure 1. Novel EPAP device 
thin bridge. Each cylindrical body is designed to fit comfortably into respective nares and remain in place during sleep. Due to the presence of a one-way valve, airflow during inhalation remains unimpeded. During expiration, however, airflow resistance is increased, allowing for pneumatic splinting to take place. Thus, airway patency and oxygenation are improved. The device was designed by Dr. Eliezer Nussbaum in 2014 and received a US Patent (\#US20160051397A1).

Following the preliminary assessment of clinical findings and PSG data obtained on the initial six subjects tested, it was determined that a large flow leak was present around the cylindrical bodies fitted into each nostril. Adjustments were made to the device, and radially projecting fins were added onto the exterior of each barrel component. Additionally, 3 holes [0.4-0.5 $\mathrm{mm}$ in diameter each] were drilled into the periphery of the interior aspect of the cylindrical holding in order to relieve excess back-up pressure.

\section{Statistical methods}

Average age and BMI were tested for between device group differences using independent t-tests Process measures of days between test points (pre and post) and device comfort level were described by median [IQR] and group differences evaluated using non-parametric Mann-Whitney $U$ test. In each group, outcomes were examined post intervention in relation to pre levels using Wilcoxen signed-rank test. Ranks were reported for each measure to indicate the number of patients whose value pre $<$ post, pre $>$ post, or unchanged (tied). Direction of change indicative of improvement was outcome specific. Change in outcome was deemed significant at the 0.05 level and indication of trend noted when significance in range of 0.05-0.10. In device group 2 (POB1-POB10), difference scores for four measures (mean $\mathrm{SpO}^{2}$, minimum $\mathrm{O}^{2} \_$Sat, \# apneas, \# obstructive apneas) did not substantially violate the assumption of normality and supplemental information was provided to describe mean (SD) with significance of change using paired t-tests. All analyses were performed using SPSS V18.0.

\section{Results}

Sixteen patients participating in the pilot study ranged in age from 40-73 years and were slightly older in the group who received device 1 compared to device $2(64.0(\mathrm{SD}=4.0)$ vs. $52.8(\mathrm{SD}=10.2), \mathrm{p}=.023)$. Average $\mathrm{BMI}$ in all 16 patients was $33.2(\mathrm{SD}=7.5)$ and similar in both device groups, $\mathrm{p}=.976 ; 68.9 \%$ were obese based on BMI $>30$. Median days elapsed between pre and post measurements of outcomes was 158 $\mathrm{d}[\mathrm{IQR}=98,210]$ and $510 \mathrm{~d}$ [IQR=295, 687] in device groups 1 and 2, respectively, $\mathrm{p}=.086$.

\section{Device 1. Patient outcomes and device comfort}

Patients in the device 1 cohort experienced a relatively high average number of arousals prior to intervention, median $=88$ [IQR 3.0, 139.0], Table 1. After use of the device, patients reported no arousals, $\mathrm{p}=.043$. Minimum $\mathrm{O} 2$ saturation improved following device use in 5 of the 6 patients, $p=.075$. An equal number of patients had a reduced as did an increased apnea indicator score following the intervention. Prompts to improve the device included 5 of 6 patients reporting more total hypopneas and higher scores on the ApneaHypopnea Index (AHI) ( $\mathrm{p}<.05)$. In addition, comfort rating of device 1 across the 6 subjects prompted modifications as values closer to 1 on 5 point scale indicate better comfort and the median score was 4.5 [IQR 2.0, 5.0].

\section{Device 2. Improved patient outcomes and device comfort}

There was noticeable improvement in comfort of device 2 as the average score was closer to the best possible ranking of 1 across the 10 patients in this group, median=2.0 [IQR 2.0, 3.0], $\mathrm{p}=.104$ ], Table 2. Although sleep efficiency was slightly lower, on average, following use of device $2(\mathrm{p}=.022)$, mean $\mathrm{O}^{2}$ saturation increased in 7 of the 10 patients $(\mathrm{p}=.057)$ and minimum $\mathrm{O}^{2}$ saturation increased in all 10 subjects $(\mathrm{p}=.005)$. Improvement across apnea indicators was significant.

Table 1. Device 1 (Patients P001-P006): change in outcome measures pre vs. post ( $\mathrm{N}=6$ patients). Patient assessment required at both time points to test significance

\begin{tabular}{|c|c|c|c|c|c|c|}
\hline & \multirow[b]{2}{*}{$\begin{array}{c}\text { Pre } \\
\text { Median [IQR] }\end{array}$} & \multirow[b]{2}{*}{$\begin{array}{c}\text { Post } \\
\text { Median [IQR] }\end{array}$} & \multicolumn{3}{|c|}{ Ranks } & \multirow[b]{2}{*}{$\begin{array}{c}\text { Difference significant, } \\
\text { p-value }^{\mathrm{a}}\end{array}$} \\
\hline & & & $\begin{array}{c}\text { N } \\
\text { Pre }<\text { Post }\end{array}$ & $\begin{array}{c}\mathrm{N} \\
\text { Pre }>\text { Post }\end{array}$ & $\begin{array}{c}\mathbf{N} \\
\text { Ties }\end{array}$ & \\
\hline \multicolumn{7}{|l|}{ Efficiency } \\
\hline Sleep Efficiency & $73.4 \%[66.0,89.7]$ & $80.8 \%[77.7,88.6]$ & 3 & 3 & 0 & $\mathrm{P}=.345$ \\
\hline Mean SpO2 & $91.4[86.3,93.2]$ & $90.4[90.4,92.6]$ & 3 & 2 & 0 & $\mathrm{P}=.500$ \\
\hline Min O2_sat & $79.0[0.0,82.0]$ & $89.4[78.0,90.5]$ & 5 & 1 & 0 & $\mathrm{P}=.075$ \\
\hline Arousals Total & $88.0[3.0,139.0]$ & 0 all six patients & 0 & 5 & 0 & $P=.043$ * \\
\hline \multicolumn{7}{|l|}{ Apnea } \\
\hline Total \# Apnea & $5.5[1.0,13.0]$ & $8.5[0.0,38.0]$ & 3 & 3 & 0 & $\mathrm{P}=.753$ \\
\hline Total \# Obstructive Apnea & $5.5[1.0,13.0]$ & $8.0[0.0,38.0]$ & 3 & 3 & 0 & $\mathrm{P}=.833$ \\
\hline Apnea Index & $1.3[0.3,2.2]$ & $1.4[0.0,4.7]$ & 3 & 3 & 0 & $\mathrm{P}=.753$ \\
\hline \multicolumn{7}{|l|}{ Hypopnea } \\
\hline Total \# Hypopneas & $38.5[25.0,86.0]$ & $100.0[29.0,188]$ & 5 & 1 & 0 & $\mathrm{P}=.046 *$ \\
\hline Hypopnea Index & $7.5[5.0,12.9]$ & $15.5[4.1,23.2]$ & 4 & 2 & 0 & $\mathrm{P}=.249$ \\
\hline \multicolumn{7}{|l|}{ AHI \& REM } \\
\hline AHI & $8.9[7.1,23.3]$ & $15.5[8.3,27.2]$ & 5 & 1 & 0 & $P=.046$ * \\
\hline Time in REM & $27.0[9.0,51.5]$ & $44.8[15.0,49.5]$ & 3 & 2 & 1 & $\mathrm{P}=.500$ \\
\hline \% Total Time in REM & $10.1[4.9,15.0]$ & $10.0[3.8,12.9]$ & 3 & 2 & 1 & $\mathrm{P}=.686$ \\
\hline $\mathrm{AHI}$ in REM & $29.1[13.6,35.6]$ & $24.2[16.4,32.0]$ & 2 & 3 & 0 & $\mathrm{P}=.500$ \\
\hline \multicolumn{7}{|l|}{ Process Measure(s) } \\
\hline Days between measures & Pre to post: & $158 \mathrm{~d}[98,210]$ & & & & \\
\hline Comfort & & $4.5[2.0,5.0]$ & & & & \\
\hline
\end{tabular}

${ }^{a}$ p-value based on Wilcoxen signed rank test

* Significant, $p<.05$ 
Table 2. Device 2 (Patients POB1-POB10): change in outcome measures pre vs. post ( $\mathrm{N}=10$ patients). Patient assessment required at both time points to test significance

\begin{tabular}{|c|c|c|c|c|c|c|}
\hline & \multirow{2}{*}{$\begin{array}{c}\text { Pre } \\
\text { Median [IQR] }\end{array}$} & \multirow{2}{*}{$\begin{array}{c}\text { Post } \\
\text { Median [IQR] }\end{array}$} & \multicolumn{3}{|c|}{ Ranks } & \multirow{2}{*}{$\begin{array}{c}\text { Difference significant, } \\
\text { p-value }\end{array}$} \\
\hline & & & $\begin{array}{c}\text { N } \\
\text { Pre }<\text { Post }\end{array}$ & $\begin{array}{c}\text { N } \\
\text { Pre }>\text { Post }\end{array}$ & $\begin{array}{c}\mathbf{N} \\
\text { Ties }\end{array}$ & \\
\hline \multicolumn{7}{|l|}{ Efficiency } \\
\hline Sleep Efficiency & $86.6 \%[83.0,93.0]$ & $76.5 \%[70.5,81.1]$ & 2 & 8 & 0 & $P=.022 *$ \\
\hline Mean SpO2 & $93.8[93.0,95.0]$ & $94.3[93.0,96.0]$ & 7 & 3 & 0 & $\mathrm{P}=.057 \sim$ \\
\hline Min O2_sat & $81.5[79.0,84.0]$ & $85.0[83.0,88.0]$ & 10 & 0 & 0 & $\mathrm{P}=.005$ * \\
\hline Arousals Total & Not assessed & Not assessed & & & & \\
\hline \multicolumn{7}{|l|}{ Apnea } \\
\hline Total \# Apnea & $11.5[0.0,21.0]$ & $1.0[0.0,7.0]$ & 2 & 6 & 2 & $\mathrm{P}=\mathbf{. 0 3 5}$ * \\
\hline Total \# Obstructive Apnea & $13.5[10.0,27.0]$ & $2.5[1.0,7.0]$ & 1 & 5 & 0 & $\mathrm{P}=.046$ * \\
\hline Apnea Index & $3.4[0.0,4.3]$ & $0.2[0.0,1.7]$ & 1 & 7 & 2 & $\mathrm{P}=.025$ * \\
\hline \multicolumn{7}{|l|}{ Hypopnea } \\
\hline Total \# Hypopneas & $31.5[13.0,42.0]$ & $20.5[13.0,36.0]$ & 4 & 5 & 1 & $\mathrm{P}=.553$ \\
\hline Hypopnea Index & $7.7[4.9,12.5]$ & $5.2[2.3,9.5]$ & 3 & 7 & 0 & $\mathrm{P}=.202$ \\
\hline \multicolumn{7}{|l|}{ AHI \& REM } \\
\hline AHI & $11.6[8.0,13.8]$ & $6.1[2.3,8.7]$ & 1 & 9 & 0 & $\mathrm{P}=.017$ * \\
\hline Time in REM & $29.3[14.5,39.5]$ & $31.7[6.0,53.0]$ & 6 & 4 & 0 & $\mathrm{P}=.721$ \\
\hline \% Total Time in REM & Not assessed & Not assessed & & & & \\
\hline AHI in REM & $27.3[16.8,44.3]$ & $23.0[11.5,29.5]$ & 0 & 3 & 0 & Inadequate data \\
\hline \multicolumn{7}{|l|}{ Process Measures } \\
\hline Days between measures & Pre to post: & $510 \mathrm{~d}[295,687]$ & & & & \\
\hline Comfort & & $2.0[2.0,3.0]$ & & & & \\
\hline
\end{tabular}

${ }^{a}$ p-value based on Wilcoxen signed rank test

* Significant, $p<.05$

$\sim$ Borderline significant, $\mathrm{p}=.05-.10$

Total number of apnea episodes, total number of obstructive apnea, and apnea index score were reduced in the majority of patients, $\mathrm{p}<.05$. Although average number of hypopnea events and the hypopnea index did not appreciably differ pre to post period, 9 of the 10 patients had less severe AHI scores following use of device $2(\mathrm{p}=.017)$.

\section{Adverse events}

There were no serious device-related adverse events associated with the use of the EPAP device in our study. Device-related adverse events resulting in study discontinuation occurred in 2 of the 19 patients tested.

\section{Discussion}

The major finding of the study was the significant decline in AHI scores compared to baseline scores, with an average $51 \%$ decrease in patients who used the most updated device. In 7 subjects who were tested originally with AHI scores in the mild to moderate range, 4 decreased into the normal range and 3 into the mild range. For all patients tested, the device demonstrated an improvement in their overall apnea hypopnea index, and thus, an improvement in sleep quality.

While there was an overall improvement in the oxygen desaturation index (ODI) scores across nearly all subjects, 2 of the 7 subjects actually had an increase in their scores. It appears there is a small improvement in the average saturation rate in our first patient population (Device 1) but without statistical significance $(p=0.075)$. Device \#2 demonstrated significant improvement in average oxygen saturation rates. There is a more notable improvement in the minimum saturation rate across all subjects, however, with a $5 \%$ overall increase across the study subjects.

Each subject served as his or her own control. Each subject had a baseline PSG which served as the control comparison to the device deployed PSG. This research design model in a pilot study provided insight for design of future studies and indication of benefit for those suffering from OSA. For some of the patients, the second device was entirely therapeutic while for others, the first device was only partially therapeutic. As such, in the clinical setting it would be beneficial to obtain PSG evaluations with the second device as a potential therapeutic modality. However, given the inadequate adherence to treatment with CPAP, even a partial reduction in the AHI would be of benefit to those are unable or unwilling to use their CPAP machines and can benefit from this new device.

In summary, this pilot study has successfully demonstrated the feasibility of using the EPAP device in patients with OSA. The device has been well tolerated by most study subjects and standard sleep variables can be acquired with the device in place. Initial findings on the most updated model indicate significant improvement in AHI scores and the minimum oxygen saturation rates. Indeed, the device offers the potential of OSA therapy with a significantly less expensive, easy to clean, disposable and portable alternative to conventional CPAP. Our pilot study incorporated a small number of patients. In order to determine the efficacy of the device, more subjects will need to undergo testing to further confirm its benefits.

\section{References}

1. Colrain IM, Brooks S, Black J (2008) A pilot evaluation of a nasal expiratory resistance device for the treatment of obstructive sleep apnea. J Clin Sleep Med 4: 426-433. [Crossref]

2. Berry RB, Kryger MH, Massie CA (2011) A novel nasal expiratory positive airway pressure device for the treatment of obstructive sleep apnea: a randomized controlled trial. Sleep 34: 479-485. [Crossref]

3. Kryger MH, Berry RB, Massie CA (2011) Long term use of a nasal expiratory positive airway pressure (EPAP) device as a treatment for obstructive sleep apnea. J Clin Sleep Med 7: 449-453. [Crossref] 
4. Rosenthal L, Massie CA, Dolan DC, Loomas B, Kram J, et al. (2009) A multicenter, prospective study of a novel nasal EPAP device in the treatment of obstructive sleep apnea: efficacy and 30-day adherence. J Clin Sleep Med 5: 532-537. [Crossref]

5. Walsh JK, Griffin KS, Forst EH, Ahmed HH, Eisenstein RD, et al. (2011) A convenient expiratory positive airway pressure nasal device for the treatment of sleep apnea in patients non-adherent with continuous positive airway pressure. Sleep Medicine 12: 147-152. [Crossref]

6. Patel AV, Hwang D, Masdeu MJ, Chen GM, Rapoport DM, et al. (2011) Predictors of response to a nasal expiratory resistor device and its potential mechanisms of action for treatment of obstructive sleep apnea. Journal of Clinical Sleep Medicine 7: 13-22. [Crossref]

7. Adams G (2011) Retrospective cases series analysis of a nasal expiratory positive airway pressure (EPAP) device to treat obstructive sleep apnea in a clinical practice. SLEEP 34: A146.

8. Riaz M, Certal V, Nigam G, Abdullatif J, Zaghi S, et al. (2015) Nasal Expiratory Positive Airway Pressure Devices (Provent) for OSA: A Systematic Review and MetaAnalysis. Sleep Disord 2015: 734798. [Crossref]
9. Epstein L, Kristo D, Strollo PJ Jr, Friedman N, Malhotra A, et al. (2009) Clinical guideline for the evaluation, management and long-term care of obstructive sleep apnea in adults. J Clin Sleep Med 5: 263-276. [Crossref]

10. Sullivan CE, Berthon-Jones M, Issa F, Eves L (1981) Reversal of obstructive sleep apnoea by continuous positive airway pressure applied through the nares. Lancet 8225: 862-865

11. Weaver TE, Grunstein RR (2008) Adherence to continuous positive airway pressure therapy: the challenge to effective treatment. Proc Am Thorac Soc 5: 173-178. [Crossref]

12. Doshi R, Westbrook P (2011) Nasal Expiratory Positive Airway Pressure (EPAP) for the treatment of obstructive sleep apnea: A review of clinical studies of Provent therapy. Resp Therapy 6: 45-49.

13. Mahadevia AK, Onal E, Lopata M (1983) Effects of expiratory positive airway pressure on sleep-induced respiratory abnormalities in patients with hypersomnia-sleep apnea syndrome. Am Rev Dis 128: 708-711. [Crossref]

Copyright: $@ 2019$ Randhawa I. This is an open-access article distributed under the terms of the Creative Commons Attribution License, which permits unrestricted use, distribution, and reproduction in any medium, provided the original author and source are credited. 